\title{
Searching efficient estimator of population variance using tri-mean and third quartile of auxiliary variable
}

\author{
S.K. Yadav* \\ Department of Statistics, \\ Babasaheb Bhimrao Ambedkar University, \\ Lucknow-226025, U.P., India \\ Email: drskystats@gmail.com \\ *Corresponding author
}

\section{Dinesh K. Sharma}

Department of Business, Management and Accounting, University of Maryland Eastern Shore,

Princess Anne, MD 21853, USA

Email: profdksharma@gmail.com

\section{S.S. Mishra}

Department of Mathematics and Statistics

(Centre of Excellence on Advanced Computing),

Dr. Ram Manohar Lohia Avadh University,

Faizabad-224001, U.P., India

Email: sant_x2003@yahoo.co.in

\begin{abstract}
This paper concerns with the estimation of population variance of study variable using tri-mean and third quartile of the auxiliary variable. In this study, the sampling properties, bias and mean squared error of the proposed estimator are demonstrated. The justification of the performance of the proposed estimator under SRSWOR has been made with reference to the competing estimators of population variance, the sample variance, Isaki (1983) estimator, the estimator due to Upadhyaya and Singh (1999), Kadilar and Cingi (2006) estimators, Subramani and Kumarapandiyan (2012) estimators, Khan and Shabbir (2013) estimator and Maqbool and Javaid (2017) estimator of population variance. Based on data provided by Murthy (1967), it has been demonstrated that the proposed estimator has shown a significant improvement over all competing estimators of population variance.
\end{abstract}

Keywords: study variable; auxiliary variable; bias; mean squared error; efficiency; tri-mean; third quartile.

Reference to this paper should be made as follows: Yadav, S.K., Sharma, D.K. and Mishra, S.S. (2019) 'Searching efficient estimator of population variance using tri-mean and third quartile of auxiliary variable', Int. J. Business and Data Analytics, Vol. 1, No. 1, pp.30-40. 
Biographical notes: S.K. Yadav is a Faculty in the Department of Statistics at the Babasaheb Bhimrao Ambedkar University Lucknow, U.P., India. He earned his MSc and $\mathrm{PhD}$ in Statistics from the Lucknow University and qualified to the National Eligibility Test. He has published 82 papers in national and international journals of repute and two books from an international publisher. $\mathrm{He}$ is a referee for 17 reputed international journals. He has been awarded Young Scientist Award in 2016 for his contribution in the field survey sampling by Venus International Research Foundation, Chennai, India. He presented papers in more than 18 national and international conferences and also delivered invited talks in several conferences.

Dinesh K. Sharma is a Professor of Quantitative Methods and Computer Applications in the Department of Business, Management and Accounting at the University of Maryland Eastern Shore. He earned his MS in Mathematics, MS in Computer Science, $\mathrm{PhD}$ in Operations Research, and a second $\mathrm{PhD}$ in Management. He has published over 90 refereed journal articles and 110 conference proceedings and has also won 12 best paper awards. Some of his work appeared in the International Journal of Computers and Applications, International Journal of Computer Mathematics, Computers and Mathematics with Applications, Journal of Applied Mathematics and Computing, International Journal of Production Economics, and International Journal of Logistics, to name a few. He is the Editor-in-Chief of the Journal of Global Information Technology and Review of Business and Technology Research.

S.S. Mishra is a Professor in the Department of Mathematics and Statistics at Dr. Ram Manohar Lohia Avadh University, Faizabad, UP, India. He received his MSc (Maths with Stats), PhD (Maths), and DSc (O.R. and Comp) degrees. $\mathrm{He}$ was selected as a Faculty under World Bank Project/UNDP at the Department of Applied Mathematics, Debub University, Ethiopia, N.E. Africa. He received a Best Research Paper Award in 2010 by GDBA Maryland and the University of Maryland E.S. sponsored conference, USA. He is also the recipients of Grant Fellowships of Int. Fed. of Information Processing, Austria and ICIAM, Switzerland. He has published more than 90 papers in national and international journals, three books from international; publishers, three research projects and 30 talks/papers in national and international conferences.

\section{Introduction}

Population variance is an indication measure of dispersion. It represents the aggregation of the random variable dispersed around its mean. Searching for efficient sampling technique from large target population is essential. The apparent estimator for any parameter is the corresponding statistics. Thus, to estimate the population variance, sample variance of the study variable is an intuitive estimator. The chosen auxiliary variable will be highly positively or negatively correlated with study variable. The auxiliary information can be used in different forms at various stages of sampling for improved estimation of population parameters (Yadav et al., 2018) and so is for population variance. 
For improving estimation of population variance of the study variable using auxiliary variables, various authors, such as Das and Tripathi (1978), Isaki (1983), Prasad and Singh (1990), Kadilar and Cingi (2006) and Singh et al. (2008) have suggested various estimators of population variance. Khan and Shabbir (2013) utilised information on the correlation coefficient between study variable and auxiliary variable and third quartile of the auxiliary variable to estimate population variance. Maqbool and Javaid (2017) proposed tri-mean and semi quartile average of auxiliary variable for the estimation of population variance of study variable. Further, for the improved estimation of the population variance, we search for even biased estimator, but having lesser MSE, which means that its sampling distribution should be very close to true the population variance of the study variable.

To achieve this goal, the adoption of the tri-mean and third quartile of the auxiliary variable has been used in this research. The auxiliary information has been introduced at estimation stage in the form of population tri-mean and third quartile. When it is highly positively correlated with study variable $(Y)$, and the line of regression of $Y$ on $X$ passes through the origin, the ratio type estimators are proposed for improved estimation of population parameters while product type estimators are considered when $X$ and $Y$ are negatively correlated. Apart from the usage of the above situation, regression estimators are used when the line of regression does not pass through the origin.

Let the population under consideration consists of $N$ distinct, independent and identifiable units. Let $\left(x_{i}, y_{i}\right), i=1,2, \ldots, n$ be a sample of size $n$ drawn on the bivariate paradigm $(X, Y)$ using a simple random sampling without replacement (SRSWOR) scheme. Let $\bar{X}$ be the population mean for auxiliary variable and $\bar{Y}$ the population mean of study variable respectively. Let $\bar{x}$ and $\bar{y}$ be the corresponding sample means of auxiliary and study variables respectively and are unbiased estimators of $\bar{X}$ and $\bar{Y}$ respectively. Let $\rho$ be the correlation coefficient between $X$ and $Y$ and $Q_{r}=Q_{3}-Q_{1}$ be the inter-quartile range, semi-quartile range $Q_{d}=\frac{Q_{3}-Q_{1}}{2}$, quartile average $Q_{a}=\frac{Q_{3}+Q_{1}}{2}$ of the auxiliary variable $X$. We propose a ratio type estimator of $\bar{Y}$ using tri-mean $T M=\left(Q_{1}+2 Q_{2}+Q_{3}\right) / 4$ and $Q_{r}$ of auxiliary variable.

Further considering many estimators in literature, Singh et al. (2011) suggested estimators for estimating population variance using auxiliary attributes. Khan and Shabbir (2013) utilised information on the correlation coefficient between study and auxiliary variable and third quartile of the auxiliary variable to estimate population variance. Subramani and Kumarapandiyan (2015) suggested some modified estimators of population variance of study variable with the use of known parameters like coefficient of variation, kurtosis, median, quartiles and deciles of auxiliary variable. Maqbool et al. (2016) proposed a modified ratio estimator using non-conventional location parameters because these parameters take care of outliers in the data. Recently, Maqbool and Javaid (2017) using tri-mean and semi quartile average of auxiliary variable, estimated the population variance of study variable. Yasmeen et al. (2018) proposed the Exponential type estimators of finite population variance using transformed auxiliary variables and shown through numerical example that their proposed estimators are better than the competing estimators of population variance. Etebong (2018) suggested a modified ratio type estimator of population variance using auxiliary information and showed improvement over other estimators both theoretically and empirically. Adichwal et al. (2018) proposed a generalised exponential ratio type estimator of population variance 
using information on auxiliary variable and shown improvement over other estimator of population variance of study variable. Ismail et al. (2018) suggested an improved generalised ratio-product type estimator of population variance using known information on two auxiliary variables. They have presented various estimators of population variance as members of their family of estimators. Both theoretically and numerically they have shown improvement over other estimators. Singh and Pal (2018) suggested a new efficient class of population variance using auxiliary attributes. They have demonstrated many estimators as a special case of their family and shown improvement over other estimators both theoretically and empirically.

In this research, we propose an estimator of population variance of study variable using tri-mean and third quartile of the auxiliary variable. The proposed estimator is an adaptation of the estimators presented by Khan and Shabbir (2013) and Maqbool and Javaid (2017). The bias and MSE of the proposed estimator are found correct up to an approximation of degree one. It is presented that the proposed estimator is more efficient than all competing estimators of population variance of study variable under simple random sampling scheme. A numerical example supports the theoretical findings.

The remainder of the paper is organised as follows: Section 2 gives a brief review of existing estimators of population variance of study variable. Section 3 proposes an improved estimator of the population variance using the known tri-mean and third quartile of the auxiliary variable. Section 4 presents the efficiency comparison with the suggested and the existing estimators. Section 5 demonstrates an empirical study under consideration. Section 6 presents the results of the study. Finally, the paper ends with conclusions in Section 7.

\section{Variance estimators}

The sample variance which is the most appropriate estimator of population variance is given by,

$$
t_{0}=s_{y}^{2}
$$

where $s_{y}^{2}=\frac{1}{n-1} \sum_{i=1}^{n}\left(y_{i}-\bar{y}\right)^{2}$

It is well established that estimator in equation (1) is unbiased for population variance of study variable, and its variance up to the first order of approximation is given by,

$$
V\left(t_{0}\right)=\lambda S_{y}^{4}\left(\lambda_{40}-1\right)
$$

where $\quad \lambda_{r s}=\frac{\mu_{r s}}{\mu_{20}^{r / 2} \mu_{02}^{s / 2}}, \quad \mu_{r s}=\frac{1}{N-1} \sum_{i=1}^{N}\left(Y_{i}-\bar{Y}\right)^{r}\left(X_{i}-\bar{X}\right)^{s}, \quad \lambda=\frac{(1-f)}{n} \quad$ and $\quad f=\frac{n}{N}$, $S_{y}^{2}=\frac{1}{N-1} \sum_{i=1}^{N}\left(y_{i}-\bar{Y}\right)^{2}, S_{y}^{4}=\left(S_{y}^{2}\right)^{2}$.

Isaki (1983) proposed the following usual ratio estimator of population variance of study variable, using positively correlated auxiliary variable as, 


$$
t_{R}=s_{y}^{2}\left[\frac{S_{x}^{2}}{s_{x}^{2}}\right]
$$

where $s_{x}^{2}=\frac{1}{n-1} \sum_{i=1}^{n}\left(x_{i}-\bar{x}\right)^{2}, \quad S_{x}^{2}=\frac{1}{N-1} \sum_{i=1}^{N}\left(X_{i}-\bar{X}\right)^{2}, \quad \bar{X}=\frac{1}{N} \sum_{i=1}^{N} X_{i}, \quad \bar{Y}=\frac{1}{N} \sum_{i=1}^{N} Y_{i}$, $\bar{x}=\frac{1}{n} \sum_{i=1}^{n} x_{i}, \bar{y}=\frac{1}{n} \sum_{i=1}^{n} y_{i}$.

The bias and MSE of above estimator correct up to approximations of degree one are respectively,

$$
\begin{aligned}
& B\left(t_{R}\right)=\lambda S_{y}^{2}\left[\left(\lambda_{04}-1\right)-\left(\lambda_{22}-1\right)\right], \\
& \operatorname{MSE}\left(t_{R}\right)=\lambda S_{y}^{4}\left[\left(\lambda_{40}-1\right)+\left(\lambda_{04}-1\right)-2\left(\lambda_{22}-1\right)\right],
\end{aligned}
$$

Upadhyaya and Singh (1999) suggested the following ratio type estimator of population variance utilising information on population coefficient of kurtosis of auxiliary variable $\left(\beta_{2(x)}\right)$ as,

$$
t_{1}=s_{y}^{2}\left[\frac{S_{x}^{2}+\beta_{2(x)}}{s_{x}^{2}+\beta_{2(x)}}\right]
$$

The bias and MSE of above estimator correct up to approximations of degree one are respectively,

$$
\begin{aligned}
& B\left(t_{1}\right)=\lambda S_{y}^{2} R_{1}\left[R_{1}\left(\lambda_{04}-1\right)-\left(\lambda_{22}-1\right)\right] \\
& \operatorname{MSE}\left(t_{1}\right)=\lambda S_{y}^{4}\left[\left(\lambda_{40}-1\right)+R_{1}^{2}\left(\lambda_{04}-1\right)-2 R_{1}\left(\lambda_{22}-1\right)\right]
\end{aligned}
$$

where $R_{1}=\frac{S_{x}^{2}}{S_{x}^{2}+\beta_{2(x)}}$.

Kadilar and Cingi (2006) suggested three ratio type estimators of population variance utilising information on population coefficient of variation $\left(C_{x}\right)$ and population coefficient of kurtosis of auxiliary variable $\left(\beta_{2(x)}\right)$ as,

$$
\begin{aligned}
& t_{2}=s_{y}^{2}\left[\frac{S_{x}^{2}+C_{x}}{s_{x}^{2}+C_{x}}\right] \\
& t_{3}=s_{y}^{2}\left[\frac{S_{x}^{2} \beta_{2(x)}+C_{x}}{s_{x}^{2} \beta_{2(x)}+C_{x}}\right] \\
& t_{4}=s_{y}^{2}\left[\frac{S_{x}^{2} C_{x}+\beta_{2(x)}}{s_{x}^{2} C_{x}+\beta_{2(x)}}\right]
\end{aligned}
$$

The biases and MSEs of above estimators correct up to approximations of degree one are respectively,

$$
B\left(t_{i}\right)=\lambda S_{y}^{2} R_{i}\left[R_{i}\left(\lambda_{04}-1\right)-\left(\lambda_{22}-1\right)\right],(i=2,3,4)
$$




$$
\operatorname{MSE}\left(t_{i}\right)=\lambda S_{y}^{4}\left[\left(\lambda_{40}-1\right)+R_{i}^{2}\left(\lambda_{04}-1\right)-2 R_{i}\left(\lambda_{22}-1\right)\right],(i=2,3,4)
$$

where $R_{2}=\frac{S_{x}^{2}}{S_{x}^{2}+C_{x}}, \quad R_{3}=\frac{S_{x}^{2} \beta_{2(x)}}{S_{x}^{2} \beta_{2(x)}+C_{x}}, \quad R_{4}=\frac{S_{x}^{2} C_{x}}{S_{x}^{2} C_{x}+\beta_{2(x)}}$.

Subramani and Kumarapandiyan (2012) by utilising information on quartiles and functions of quartiles of auxiliary variable, suggested the following ratio type estimators of population variance as,

$$
\begin{aligned}
& t_{5}=s_{y}^{2}\left[\frac{S_{x}^{2}+Q_{1}}{s_{x}^{2}+Q_{1}}\right] \\
& t_{6}=s_{y}^{2}\left[\frac{S_{x}^{2}+Q_{3}}{s_{x}^{2}+Q_{3}}\right] \\
& t_{7}=s_{y}^{2}\left[\frac{S_{x}^{2}+Q_{r}}{s_{x}^{2}+Q_{r}}\right] \\
& t_{8}=s_{y}^{2}\left[\frac{S_{x}^{2}+Q_{d}}{s_{x}^{2}+Q_{d}}\right] \\
& t_{9}=s_{y}^{2}\left[\frac{S_{x}^{2}+Q_{a}}{s_{x}^{2}+Q_{a}}\right]
\end{aligned}
$$

The biases and MSEs of above estimators correct up to approximations of degree one are respectively,

$$
\begin{aligned}
& B\left(t_{i}\right)=\lambda S_{y}^{2} R_{i}\left[R_{i}\left(\lambda_{04}-1\right)-\left(\lambda_{22}-1\right)\right],(i=5,6,7,8,9) \\
& \operatorname{MSE}\left(t_{i}\right)=\lambda S_{y}^{4}\left[\left(\lambda_{40}-1\right)+R_{i}^{2}\left(\lambda_{04}-1\right)-2 R_{i}\left(\lambda_{22}-1\right)\right],(i=5,6,7,8,9) \\
& R_{5}=\frac{S_{x}^{2}}{S_{x}^{2}+Q_{1}}, R_{6}=\frac{S_{x}^{2}}{S_{x}^{2}+Q_{3}}, R_{7}=\frac{S_{x}^{2}}{S_{x}^{2}+Q_{r}}, R_{8}=\frac{S_{x}^{2}}{S_{x}^{2}+Q_{d}}, R_{9}=\frac{S_{x}^{2}}{S_{x}^{2}+Q_{a}} .
\end{aligned}
$$

Khan and Shabbir (2013) utilised information on correlation coefficient between study and auxiliary variable and third quartile of the auxiliary variable and proposed the following estimator as,

$$
t_{10}=s_{y}^{2}\left[\frac{S_{x}^{2} \rho+Q_{3}}{s_{x}^{2} \rho+Q_{3}}\right]
$$

The bias and MSE of above estimator correct up to approximations of degree one are respectively,

$$
\begin{aligned}
& B\left(t_{10}\right)=\lambda S_{y}^{2} R_{10}\left[R_{10}\left(\lambda_{04}-1\right)-\left(\lambda_{22}-1\right)\right] \\
& \operatorname{MSE}\left(t_{10}\right)=\lambda S_{y}^{4}\left[\left(\lambda_{40}-1\right)+R_{10}^{2}\left(\lambda_{04}-1\right)-2 R_{10}\left(\lambda_{22}-1\right)\right]
\end{aligned}
$$




$$
R_{10}=\frac{S_{x}^{2} \rho}{S_{x}^{2} \rho+Q_{3}} .
$$

Maqbool and Javaid (2017) using tri-mean and semi quartile average of auxiliary variable, proposed the following estimator as,

$$
t_{11}=s_{y}^{2}\left[\frac{S_{x}^{2}+\left(T M+Q_{a}\right)}{s_{x}^{2}+\left(T M+Q_{a}\right)}\right]
$$

The bias and MSE of above estimator correct up to approximations of degree one are respectively,

$$
\begin{aligned}
& B\left(t_{11}\right)=\lambda S_{y}^{2} R_{11}\left[R_{11}\left(\lambda_{04}-1\right)-\left(\lambda_{22}-1\right)\right] \\
& \operatorname{MSE}\left(t_{11}\right)=\lambda S_{y}^{4}\left[\left(\lambda_{40}-1\right)+R_{11}^{2}\left(\lambda_{04}-1\right)-2 R_{11}\left(\lambda_{22}-1\right)\right] \\
& R_{11}=\frac{S_{x}^{2}}{S_{x}^{2}+\left(T M+Q_{a}\right)}
\end{aligned}
$$

Various improved estimators of population variance using auxiliary information have been introduced. The recent references can be from Subramani and Kumarapandiyan (2012, 2013), Yadav and Kadilar (2013a, 2013b), Yadav and Mishra (2015), Yadav et al. (2016), Singh et al. (2016) and Abid et al. (2016).

\section{Proposed estimator}

We propose a modified ratio type estimator of population variance, motivated by Khan and Shabbir (2013) and Maqbool and Javaid (2017), using tri-mean and third quartile of the auxiliary variable as,

$$
t_{p}=s_{y}^{2}\left[\frac{S_{x}^{2}\left(T M+Q_{3}\right)}{s_{x}^{2}\left(T M+Q_{3}\right)}\right],
$$

The sampling properties of the proposed estimator, $t_{p}$ are studied using following assumptions, $s_{y}^{2}=S_{y}^{2}\left(1+\varepsilon_{0}\right)$ and $s_{x}^{2}=S_{x}^{2}\left(1+\varepsilon_{1}\right)$ such that $E\left(\varepsilon_{i}\right)=0$ for $(i=0,1)$ and $E\left(\varepsilon_{0}^{2}\right)=\frac{1-f}{n}\left(\lambda_{40}-1\right), S_{x}^{2}=S_{x}^{2}\left(1+\varepsilon_{1}\right), \quad E\left(\varepsilon_{0} \varepsilon_{1}\right)=\frac{1-f}{n}\left(\lambda_{22}-1\right)$.

Expressing $t_{p}$ in terms of $\varepsilon_{i}$ 's $(i=0,1)$, we have

$$
t_{p}=S_{y}^{2}\left(1+e_{0}\right)\left(1+R_{p} e_{1}\right)^{-1}
$$

where $R_{p}=\frac{S_{x}^{2}}{S_{x}^{2}+\left(T M+Q_{3}\right)}$.

Expanding term on right-hand side, simplifying and retaining the terms up to the first degree of approximation, we have,

$$
t_{p}=S_{y}^{2}\left(1+e_{0}-R_{p} e_{1}-R_{p} e_{0} e_{1}+R_{p}^{2} e_{1}^{2}\right)
$$


Subtracting $S_{y}^{2}$ on both the sides, we get,

$$
t_{p}-S_{y}^{2}=S_{y}^{2}\left(e_{0}-R_{p} e_{1}-R_{p} e_{0} e_{1}+R_{p}^{2} e_{1}^{2}\right)
$$

Taking expectation on both sides of (28), we get the bias of proposed estimator $t_{p}$ as,

$$
B\left(t_{p}\right)=\lambda S_{y}^{2}\left[R_{p}^{2}\left(\lambda_{04}-1\right)-R_{p}\left(\lambda_{22}-1\right)\right]
$$

Squaring both sides of equation (28), simplifying up to the first degree of approximation and taking expectation on both sides, we get the $M S E$ of the proposed estimator as,

$$
\operatorname{MSE}\left(t_{p}\right)=\lambda S_{y}^{4}\left[\left(\lambda_{40}-1\right)+R_{p}^{2}\left(\lambda_{04}-1\right)-2 R_{p}\left(\lambda_{22}-1\right)\right]
$$

\section{Efficiency comparison}

The suggested estimator in equation (27) is better than the usual mean per unit estimator in equation (1) if,

$$
\operatorname{MSE}\left(t_{p}\right)-V\left(t_{0}\right)<0, \text { if } R_{p}\left(\lambda_{04}-1\right)<2\left(\lambda_{22}-1\right)
$$

The suggested estimator in equation (27) is better than the usual ratio estimator of Isaki (1983) if,

$$
\operatorname{MSE}\left(t_{p}\right)-\operatorname{MSE}\left(t_{R}\right)<0, \text { if }\left(R_{p}+1\right)\left(\lambda_{04}-1\right)<2\left(\lambda_{22}-1\right)
$$

The suggested estimator in equation (27) is best among other competing estimators making use of auxiliary information in the form of various parameters of the auxiliary variable if,

$$
\operatorname{MSE}\left(t_{p}\right)-\operatorname{MSE}\left(t_{i}\right)<0,(i=1,2, \ldots, 10) \text { if }\left(R_{p}+R_{i}\right)\left(\lambda_{04}-1\right)<2\left(\lambda_{22}-1\right)
$$

\section{Numerical illustration}

To justify the performance of the proposed estimator and the competing estimators of population variance of study variable under SRSWOR, we have used the data from Murthy (1967) on page 228. In the numerical example, the fixed capital is the auxiliary variable $X$ and output of 80 factories is the study variable $Y$. The numerical values of the biases and the mean squared errors for the proposed and competing estimators have been computed for this data. The parameters for this data are given below,

$$
\begin{aligned}
& N=80, n=20, \bar{Y}=51.8264, \bar{X}=11.2646, \rho=0.9413, S_{y}=18.3549, \\
& C_{y}=0.3542, S_{x}=8.4563, C_{x}=0.7507, \lambda_{04}=2.8664, \lambda_{40}=2.2667, \\
& \lambda_{22}=2.2209, Q_{1}=5.1500, Q_{3}=16.975, Q_{r}=11.825, Q_{d}=5.9125, \\
& Q_{a}=11.0625 .
\end{aligned}
$$

The obtained results for proposed and competing estimators are represented in following Table 1 in the form of bias and mean squared error. 
Table 1 Bias and MSE of proposed and competing estimators of population variance

\begin{tabular}{lcc}
\hline Estimator & Bias & MSE \\
\hline Mean per unit estimator $t_{0}$ & 0 & $5,393.89$ \\
Isaki (1983) estimator $t_{R}$ & 10.87 & $3,925.16$ \\
Upadhyaya and Singh (1999) $t_{1}$ & 9.29 & $3,658.41$ \\
Kadilar and Cingi (2006) $t_{2}$ & 10.44 & $3,850.16$ \\
Kadilar and Cingi (2006) $t_{3}$ & 10.72 & $3,898.56$ \\
Kadilar and Cingi (2006) $t_{4}$ & 8.81 & $3,580.83$ \\
Subramani and Kumarapandiyan (2012) $t_{5}$ & 8.17 & $3,480.55$ \\
Subramani and Kumarapandiyan (2012) $t_{6}$ & 3.91 & $2,908.65$ \\
Subramani and Kumarapandiyan (2012) $t_{7}$ & 5.50 & $3,098.41$ \\
Subramani and Kumarapandiyan (2012) $t_{8}$ & 7.82 & $3,427.19$ \\
Subramani and Kumarapandiyan (2012) $t_{9}$ & 5.77 & $3,133.33$ \\
Khan and Shabbir (2013) $t_{10}$ & 3.62 & $2,878.56$ \\
Maqbool and Javaid (2017) $t_{11}$ & 3.03 & $2,820.06$ \\
Yadav et al. (Proposed) $t_{p}$ & 1.33 & $2,040.12$ \\
\hline
\end{tabular}

\section{Results}

From Table 1, it shows that the variance of mean per unit estimator is 5,393.89 which makes no use of auxiliary information. All the competing estimators of the population mean using auxiliary information have their MSE ranging from 2,820.06 to 3,925.16 showing that all are improved over mean per unit estimator. Among all competing estimators that make use of auxiliary information, the estimator of Maqbool and Javaid (2017) has least MSE of 2,820.06. As per our aim, it is further to be mentioned that the proposed estimator has MSE of 2,040.12, which is least among the class of all competing estimators using without and with auxiliary information of population variance of study variable under simple random sampling scheme.

\section{Conclusions}

In this paper, a ratio type estimator of population variance of study variable using information on tri-mean and the third quartile of the auxiliary variable has been suggested. The sampling properties that is bias and MSE of proposed estimator are derived correctly up to an approximation of degree one. The theoretical comparison of the proposed estimator is made with the competing estimators of population variance. The purpose of the estimation of the population parameter is to search for an estimator even biased, but its sampling distribution must be very close to the actual value of the parameter under study and so is for the population variance. The theoretical findings have been justified with the help of a dataset. Table 1, shows that the proposed estimator is best among all competing estimators of population variance under simple random sampling scheme as it has least bias and MSE among the class of all mentioned 
competing estimator of population variance. Thus the scatteredness of the numerical values of the proposed estimator shows that its sampling distribution is very close to the real value of the population variance, since it has least mean squared error among all competing estimators of population variance. Therefore, it is expected to use the proposed estimator for improved estimation of population variance of study variable with auxiliary information.

\section{Acknowledgements}

The authors are thankful to the anonymous reviewers for their valuable and helpful suggestions.

\section{References}

Abid, M., Abbas, N. Sherwani, R.A.K. and Nazir, H.Z. (2016) 'Improved ratio estimators for the population mean using non-conventional measure of dispersion', Pakistan Journal of Statistics and Operations Research, Vol. 12, No. 2, pp.353-367.

Adichwal, N.K., Kumar, J. and Singh, R. (2018) 'An improved generalized class of estimators for population variance using auxiliary variables', Cogent Mathematics \& Statistics, Vol. 5, No. 1, p.1454579.

Das, A.K. and Tripathi, T.P. (1978) 'Use of auxiliary information in estimating the finite population variance', Sankhya, Vol. 40, pp.139-148.

Etebong, P.C. (2018) 'Improved family of ratio estimators of finite population variance in stratified random sampling', Biostat Biometrics Open Acc. J., Vol. 5, No. 2, p.555659, DOI: 10.19080/ BBOAJ.2018.04.555659.

Isaki, C.T. (1983) 'Variance estimation using auxiliary information', Journal of American Statistical Association, Vol. 78, pp.117-123.

Ismail, M., Kanwal, N. and Shahbaz, M.Q. (2018) 'Generalized ratio-product-type estimator for variance using auxiliary information in simple random sampling', Kuwait Journal of Science, Vol. 45, No. 1, pp.79-88.

Kadilar, C. and Cingi, H. (2006) 'Improvement in variance estimation using auxiliary information Hacettepe', Journal of mathematics and Statistics, Vol. 35, pp.111-115.

Khan, M. and Shabbir, J. (2013) 'A ratio type estimator for the estimation of population variance using quartiles of an auxiliary variable', Journal of Statistics Applications \& Probability, Vol. 2, No. 3, pp.319-325.

Maqbool, S. and Javaid, S. (2017) 'Variance estimation using linear combination of tri-mean and quartile average', American Journal of Biological and Environmental Statistics, Vol. 3, No. 1, pp.5-9.

Maqbool, S., Raja, T.A. and Javaid, S. (2016) 'Generalized modified ratio estimator using non-conventional location parameter', Int. J. Agricult. Stat. Sci., Vol. 12, No. 1, pp.95-97.

Murthy, M.N. (1967) Sampling Theory and Methods, Statistical Publishing Society Calcutta, India.

Prasad, B. and Singh, H.P. (1990) 'Some improved ratio type estimators of finite population variance in sample surveys', Communication in Statistics: Theory and Methods, Vol. 19, pp.1127-1139.

Singh, B.K., Chanu, W.W. and Tato, Y. (2016) 'A class of ratio cum dual to ratio estimator in presence of non-response using two phase sampling scheme', Gazi University Journal of Science, Vol. 29, No. 1, pp.213-221. 
Singh, H.P. and Pal, S.K. (2018) 'An efficient new class of estimators of population variance using information on auxiliary attribute in sample surveys', Hacettepe Journal of Mathematics and Statistics, Vol. 47, No. 1, pp.267-277.

Singh, R., Kumar, M., Singh, A. and Smarandache, F. (2011) 'A family of estimators of population variance using information on auxiliary attribute', Studies in Sampling Techniques and Time Series Analysis, pp.63-70, Zip Publishing, Columbus, USA.

Singh, R., Chauhan, P., Sawan, N. and Smarandache, F. (2008) 'Ratio estimators in simple random sampling using information on auxiliary attribute', Pakistan Journal of Statistics and Operation Research, Vol. 4, No. 1, pp.47-53.

Subramani, J. and Kumarapandiyan, G. (2012) 'Variance estimation using quartiles and their functions of an auxiliary variable', International Journal of Statistics and Applications, Vol. 2, No. 5, pp.67-72.

Subramani, J. and Kumarapandiyan, G. (2013) 'A new modified ratio estimator of population mean when median of the auxiliary variable is known', Pakistan Journal of Statistics and Operation Research, Vol. 9, No. 2, pp.137-145.

Subramani, J. and Kumarapandiyan, G. (2015) 'A class of modified ratio estimators for estimation of population variance', Journal of Applied Mathematics, Statistics and Informatics, Vol. 11, No. 1, pp.91-114.

Upadhyaya, L.N. and Singh, H.P. (1999) 'Use of transformed auxiliary variable in estimating the finite population mean', Biometrical Journal, Vol. 41, No. 5, pp.627-636.

Yadav, S.K, Gupta, S., Mishra, S.S. and Shukla, A.K. (2016) 'Modified ratio and product estimators for estimating population mean in two-phase sampling', American Journal of Operational Research, Vol. 6, No. 3, pp.61-68.

Yadav, S.K. and Kadilar, C. (2013a) 'Improved class of ratio and product estimators', Applied Mathematics and Computation, Vol. 219, pp.10726-10731.

Yadav, S.K. and Kadilar, C. (2013b) 'Efficient family of exponential estimator for population mean', Hacettepe Journal of Mathematics and Statistics, Vol. 42, No. 6, pp.671-677.

Yadav, S.K. and Mishra, S.S. (2015) 'Developing improved predictive estimator for finite population mean using auxiliary information', Statistika, Vol. 95, No. 1, pp.76-85.

Yadav, S.K., Sharma, D.K., Mishra, S.S. and Shukla, A. (2018) 'Use of auxiliary variables in searching efficient estimator of population mean', International Journal of Multivariate Data Analysis, Vol. 1, No. 3, pp.230-244.

Yasmeen, U., Noor-ul-Ameem, M. and Hanif, M. (2018) 'Exponential estimators of finite population variance using transformed auxiliary variables', Proceedings of the National Academy of Sciences, India Section A: Physical Sciences, DOI: 10.1007/s40010-017-0410-5. 